\title{
WAVE PROPAGATION IN A LAYERED ELASTIC PLATE
}

\author{
R. A. SCOTT \\ Engineering Mechanics Department, University of Michigan, Michigan 48104
}

\begin{abstract}
Formal solutions to a broad class of transient, symmetric, elastic wave problems involving a three layered plate are presented in terms of the modes of harmonic wave propagation. An approximation to the lowest mode is developed and, using this and the formal solutions, far-field expressions are developed for three specific problems. These approximate expressions are analyzed numerically for two specific cases.
\end{abstract}

\section{INTRODUCTION}

IN A recent work [1], the author treated certain aspects of transient elastic wave propagation in an infinite inhomogeneous plate. There the material properties were taken to vary continuously in the thickness direction. The current paper extends that work to situations where discrete layering occurs.

Elastic transients in layered media have attracted attention over the years due to their importance in the fields of seismology and laminated composites. In seismology, considerable work has been done on the case of a single layer over a half space (see Newlands [2], Pekeris et al. [3, 4], Mitra [5], Laster et al. [6]). In the case of two layers over a half space, the work of Mitra [7] on $S H$-transients should be noted. The studies on composites are somewhat different in that most authors confine their attention to infinite periodic media, i.e. to a two-layered structure that repeats itself indefinitely. Voelker and Achenbach [8] presented approximate and exact results for transients generated by a time-dependent body force in such a medium. Peck and Gurtman [9] studied transients in a half space, the material of which was periodic. They gave and analyzed a long wave approximation, valid for the far-field (the head of the pulse approximation). In the same area, the work of Sve [10] using the effective stiffness theory of Achenbach et al. [11] should be noted.

Here, formal solutions are given to a broad class of symmetric, plane-strain, transient, wave propagation problems involving a three layered plate. The solution technique, which hinges on the modes of harmonic wave propagation, is similar to that used in [1] and is related to the methods used by the author [12], Rosenfeld and Miklowitz [13], Achenbach [14, 15], Reismann [16], Lee and Reismann [17], Wu and Plunkett [18] and Cobble [19]. The items necessary for the head of the pulse approximation are developed and then the approximation is applied to three specific problems, namely the longitudinal impact and mixed pressure shock problems for a semi-infinite plate and the sudden, symmetric application of normal line loads to an infinite plate.

\section{MODES OF HARMONIC WAVE PROPAGATION}

As in [1], solutions to transient problems will be sought in terms of the modes of harmonic wave propagation for an infinite plate. As opposed to [1] though, now the scheme is 
one of convenience rather than necessity in that, in principle, the modes can be found analytically. However, the current method leads to head of the pulse approximations more rapidly. $\dagger$

The plate geometry and the coordinates used are shown in Fig. 1. This geometry is equivalent, for the inputs used here, to two perfectly bonded elastic layers of thickness $h_{1}$ and $h_{2}-h_{1}$, respectively, in lubricated contact with a rigid half-space. In the sequel, the various problems will be examined in this latter context. The stress equations of motion are, in the absence of body forces, and assuming plane-strain conditions,

$$
\begin{array}{ll}
\frac{\partial_{j} \sigma_{x x}}{\partial x}+\frac{\partial_{j} \sigma_{x z}}{\partial z}=\rho_{j} \frac{\partial^{2}{ }_{j} u_{x}}{\partial t^{2}}, & j=1,2, \\
\frac{\partial_{j} \sigma_{x z}}{\partial x}+\frac{\partial_{j} \sigma_{z z}}{\partial z}=\rho_{j} \frac{\partial^{2}{ }_{j} u_{z}}{\partial t^{2}}, & j=1,2,
\end{array}
$$

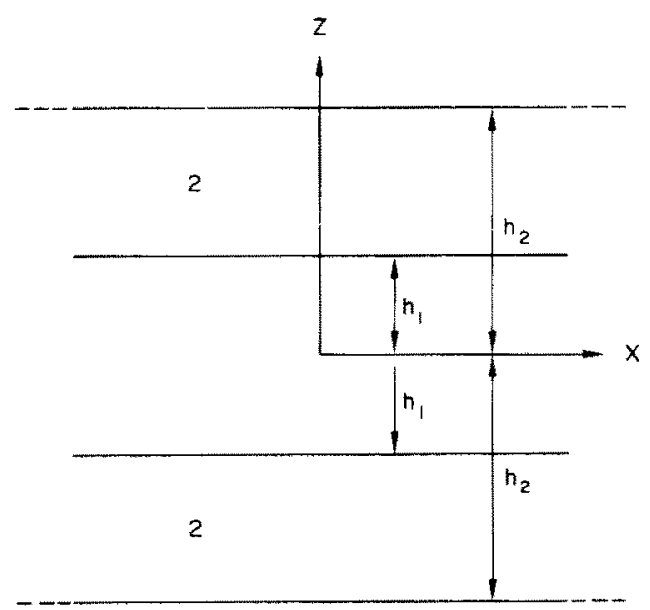

FIG. 1. Geometry of the layered plate.

where $\rho$ denotes material density, $\sigma$ stress, $u$ displacement and the subscript $j$ the layer in question. The stress-displacement relations are

$$
\begin{array}{ll}
{ }_{j} \sigma_{x x}=\lambda_{j}\left(\frac{\partial_{j} u_{x}}{\partial x}+\frac{\partial_{j} u_{z}}{\partial z}\right)+2 \mu_{j} \frac{\partial_{j} u_{x}}{\partial x}, & j=1,2, \\
{ }_{j} \sigma_{z z}=\lambda_{j}\left(\frac{\partial_{j} u_{x}}{\partial x}+\frac{\partial_{j} u_{z}}{\partial z}\right)+2 \mu_{j} \frac{\partial_{j} u_{z}}{\partial z}, & j=1,2, \\
{ }_{j} \sigma_{x z}=\mu_{j}\left(\frac{\partial_{j} u_{x}}{\partial z}+\frac{\partial_{j} u_{z}}{\partial x}\right), & j=1,2,
\end{array}
$$


where $\lambda$ and $\mu$ are Lamé's constants. The boundary conditions are

$$
\begin{aligned}
& { }_{2} \sigma_{z z}=0, \quad{ }_{2} \sigma_{x z}=0, \quad z=h_{2} \\
& { }_{1} \sigma_{x z}=0, \quad{ }_{1} u_{z}=0, \quad z=0 \text {. }
\end{aligned}
$$

Also, the assumption of a perfect bond requires the displacements and stresses to be continuous at $z=h_{1}$.

To obtain the modes of harmonic wave propagation, one sets

$$
\begin{array}{ll}
{ }_{j} u_{x}(x, z, t)={ }_{j} u_{x}^{N}(z) \mathrm{e}^{i k x+p_{N} t}, & j=1,2, \\
{ }_{j} u_{z}(x, z, t)={ }_{j} u_{z}^{N}(z) \mathrm{e}^{i k x+p_{N} t}, & j=1,2,
\end{array}
$$

where $k$ denotes wave number, $p_{N}$ a frequency like term and the index $N$ stands for the fact that an infinite number of modes exist. Substituting (8) and (9) into (3)-(5), yields modal stress-displacement relations. Typical ones are

$$
\sigma_{x x}^{N}=i k\left(\lambda_{j}+2 \mu_{j}\right)_{j} u_{x}^{N}+\lambda_{j} \frac{\mathrm{d}_{j} u_{z}^{N}}{\mathrm{~d} z}, \quad j=1,2 .
$$

Substituting the modal stress-displacement relations into (1) and (2), gives the modal equations of motion, typical ones being

$$
\frac{\mathrm{d}_{j} \sigma_{x z}^{N}}{\mathrm{~d} z}+i k_{j} \sigma_{x x}^{N}=\rho_{j} p_{N j}^{2} u_{x}^{N}, \quad j=1,2
$$

From (6) and (7), the modal boundary conditions are

$$
\begin{array}{lll}
{ }_{2} \sigma_{z z}^{N}=0, & { }_{2} \sigma_{x z}^{N}=0, & z=h_{2} \\
{ }_{1} \sigma_{x z}^{N}=0, & { }_{1} u_{z}^{N}=0, & z=0 .
\end{array}
$$

Also, the modal displacements and stresses must be continuous at $z=h_{1}$.

To develop pertinent properties of the modes the following Hermitian bilinear forms are introduced :

$$
\begin{gathered}
T\left(u^{N}, u^{M}\right)=2 \rho_{1} \int_{0}^{h_{1}}\left({ }_{1} u_{z}^{N}{ }_{1} u_{z}^{M^{*}}+{ }_{1} u_{x}^{N}{ }_{1} u_{x}^{M^{*}}\right) \mathrm{d} z+2 \rho_{2} \int_{h_{1}}^{h_{2}} \\
\quad \times\left({ }_{2} u_{z}^{N}{ }_{2} u_{z}^{M^{*}}+{ }_{2} u_{x 2}^{N} u_{x}^{M^{*}}\right) \mathrm{d} z \\
V\left(u^{N}, u^{M}\right)=2 \int_{0}^{h_{1}}\left[\frac{\mathrm{d}_{1} u_{z}^{N}}{\mathrm{~d} z}{ }_{1} \sigma_{z z}^{M^{*}}+\frac{\mathrm{d}_{1} u_{x}^{N}}{\mathrm{~d} z}{ }_{1} \sigma_{x z}^{M^{*}}+i k\right. \\
\left.\times\left({ }_{1} u_{x}^{N} \sigma_{x x} \sigma_{x{ }_{1}}^{M^{*}}+{ }_{1} u_{z}^{N}{ }_{1} \sigma_{x z}^{M^{*}}\right)\right] \mathrm{d} z+2 \int_{h_{1}}^{h_{2}}\left[\frac{\mathrm{d}_{2} u_{z}^{N}}{\mathrm{~d} z}{ }_{2} \sigma_{z z}^{M^{*}}\right. \\
\left.+\frac{\mathrm{d}_{2} u_{x}^{N}}{\mathrm{~d} z}{ }_{2} \sigma_{x z}^{M^{*}}+i k\left({ }_{2} u_{x 2}^{N} \sigma_{x x} \sigma_{x}^{M^{*}}+{ }_{2} u_{z}^{N}{ }_{2} \sigma_{x z} M^{*}\right)\right] \mathrm{d} z
\end{gathered}
$$

where ${ }^{*}$ denotes complex conjugate. Using integration by parts, equations such as (10) (13) and the continuity conditions at $z=h_{1}$, it can be shown that

$$
V\left(u^{N}, u^{M}\right)=-p_{M}^{2 *} T\left(u^{N}, u^{M}\right)
$$


Using (16) and the Hermitian properties and taking complex conjugates, it can be shown that, on defining

$$
T\left(u^{N}, u^{N}\right)=1
$$

then

$$
T\left(u^{N}, u^{M}\right)=\delta_{N M}
$$

where $\delta_{N M}$ denotes the Kronecker delta.

\section{FORMAL SOLUTIONS TO TRANSIENT PROBLEMS}

Attention will now be focused on a class of transient problems. Taking the Laplace transform and the Fourier transform defined by ${ }^{\dagger}$

$$
\tilde{f}(k)=\int_{0}^{\infty} f(x) \mathrm{e}^{-i k x} \mathrm{~d} x=\tilde{f}^{c}-i \tilde{f}^{s}
$$

where $C$ and $S$ denote cosine and sine transforms, respectively, of (1) and (2), gives the transformed equations of motion. Typical ones are

$$
\frac{\mathrm{d}_{j} \tilde{\sigma}_{x z}}{\mathrm{~d} z}+i k_{j} \tilde{\sigma}_{x x}-\left.{ }_{j} \bar{\sigma}_{x x}\right|_{x=0}=\rho_{j} p_{j}^{2} \tilde{u}_{x}, \quad j=1,2,
$$

where the bar denotes the Laplace transform, parameter $p$. Taking Laplace and Fourier transforms of (3)-(5) yields the transformed stress-displacement relations, typical ones being

$$
{ }_{j} \tilde{\sigma}_{x x}=\lambda_{j} \frac{\mathrm{d}_{j} \tilde{\bar{u}}_{z}}{\mathrm{~d} z}+i k\left(\lambda_{j}+2 \mu_{j}\right)_{j} \tilde{\tilde{u}}_{x}-\left.\left(\lambda_{j}+2 \mu_{j}\right)_{j} \bar{u}_{x}\right|_{x=0}, \quad j=1,2 .
$$

The quadruplets of modal displacements $\left({ }_{1} u_{x}^{N},{ }_{2} u_{x}^{N},{ }_{1} u_{z}^{N},{ }_{2} u_{z}^{N}\right), N=1,2, \ldots$, can be interpreted as the characteristic vectors in a vector space consisting of quadruplets of functions. Then, assuming completeness, arbitrary quadruplets can be expressed in terms of the modal displacements. In particular, the quadruplet of transformed displacements arising in transient problems can be expanded in this fashion. One obtains

$$
\begin{array}{rlrl}
j & \tilde{\tilde{u}}_{x}=\sum_{N} \Gamma_{N j} u_{x}^{N}, & j=1,2, \\
\tilde{\tilde{u}}_{z}=\sum_{N} \Gamma_{N j} u_{z}^{N}, & j=1,2,
\end{array}
$$

where the $\Gamma_{N}$ are expansion coefficients.

It can be shown, using (14), (17), (18), (22) and (23), that

$$
\Gamma_{N}=T\left(\tilde{u}, u^{N}\right) \text {. }
$$

Using the bilinear form $V\left(\tilde{u}, u^{N}\right)$, integration by parts, equations such as (20) and (21), the continuity conditions at $z=h_{1}$ and the modal boundary conditions, it can be shown that

$$
V\left(\tilde{u}, u^{M}\right)=-p_{M}^{2 *} T\left(\tilde{u}, u^{M}\right)
$$

† Later, by considering only the odd or even parts of the functions of $k$ that arise, this definition allows one to handle the longitudinal impact and pressure shock problems simultaneously. 
where $V\left(\tilde{u}, u^{M}\right)$ is given by (15), with $u^{N}$ replaced by $\tilde{u}$. To proceed further, it must be noted that

$$
\begin{aligned}
V\left(u^{N}, \tilde{u}\right)= & 2 \int_{0}^{h_{1}}\left[\frac{\mathrm{d}_{1} u_{z}^{N}}{\mathrm{~d} z}\left({ }_{1} \tilde{\sigma}_{z z}^{*}\right)_{v}+\frac{\mathrm{d}_{1} u_{x}^{N}}{\mathrm{~d} z}\left({ }_{1} \tilde{\sigma}_{x z}^{*}\right)_{v}+i k_{1} u_{x}^{N}\left({ }_{1} \tilde{\sigma}_{x x}^{*}\right)_{v}\right. \\
& \left.+i k_{1} u_{z}^{N}\left({ }_{1} \tilde{\sigma}_{x z}^{*}\right)_{v}\right] \mathrm{d} z \\
& +2 \int_{h_{1}}^{h_{2}}\left[\frac{\mathrm{d}_{2} u_{z}^{N}}{\mathrm{~d} z}\left({ }_{2} \tilde{\tilde{\sigma}}_{z z}^{*}\right)_{v}+\frac{\mathrm{d}_{2} u_{x}^{N}}{\mathrm{~d} z}\left({ }_{2} \tilde{\tilde{\sigma}}_{x z}^{*}\right)_{v}\right. \\
& \left.+i k_{2} u_{x}^{N}\left({ }_{2} \tilde{\sigma}_{x x}^{*}\right)_{v}+i k_{2} u_{z}^{N}\left(2_{2} \tilde{\tilde{\sigma}}_{x z}^{*}\right)_{v}\right] \mathrm{d} z
\end{aligned}
$$

where the subscript $v$ denotes the fact that the transformed item in question is for an infinite plate (the geometry for which the properties of $T$ and $V$ were developed), in contrast to the actual transformed items for a semi-infinite plate, denoted in the sequel by a subscript $P$. The two are related, typical relationships being

$$
\left({ }_{j} \tilde{\bar{\sigma}}_{z z}\right)_{v}=\left({ }_{j} \tilde{\bar{\sigma}}_{z z}\right)_{P}+\left.\lambda_{j j} \bar{u}_{x}\right|_{x=0}, \quad j=1,2 .
$$

Using equations such as (20), (21) and (27), integration by parts and the continuity conditions at $z=h_{1}$, it can be shown that (26) leads to

$$
V\left(u^{N}, \tilde{u}\right)=B_{h N}+\bar{B}_{O N}-p^{2 *} T\left(u^{N}, \tilde{u}\right)
$$

where

$$
\begin{gathered}
B_{h N}=\left.2\left[{ }_{2} u_{z}^{N}\left({ }_{2} \tilde{\sigma}_{z z}^{*}\right)_{P}+{ }_{2} u_{x}^{N}\left({ }_{2} \tilde{\sigma}_{x z}^{*}\right){ }_{P}\right]\right|_{z=h_{2}} \\
\bar{B}_{O N}=2 \int_{0}^{h_{1}}\left[\left.{ }_{1} \sigma_{x x 1}^{N} \bar{u}_{x}^{*}\right|_{x=0}+\left.{ }_{1} \sigma_{x z}^{N} \bar{u}_{z}^{*}\right|_{x=0}-\left.{ }_{1} u_{z}^{N} \bar{\sigma}_{x z}^{*}\right|_{x=0}\right. \\
\left.-\left.{ }_{1} u_{x 1}^{N} \bar{\sigma}_{x x}^{*}\right|_{x=0}\right] \mathrm{d} z+2 \int_{h_{1}}^{h_{2}}\left[\left.{ }_{2} \sigma_{x x 2}^{N} \bar{u}_{x}^{*}\right|_{x=0}+\left.{ }_{2} \sigma_{x z}^{N} \bar{u}_{z}^{*}\right|_{x=0}\right. \\
\left.-\left.{ }_{2} u_{z}^{N} \bar{\sigma}_{x z}^{*}\right|_{x=0}-{ }_{2} u_{x 2}^{N} \bar{\sigma}_{\left.x x\right|_{x}=0}^{*}\right] \mathrm{d} z .
\end{gathered}
$$

For problems involving an infinite plate, $B_{O N}$ is set equal to zero. Using (24), (25) and (28), and the Hermitian properties of the bilinear forms, it can be shown that

$$
\left(p^{2}-p_{N}^{2^{*}}\right) \Gamma_{N}=B_{h N}^{*}+\bar{B}_{O N}^{*} .
$$

Equations (22),(23), (29)-(31), constitute the formal solutions to a broad class of transient, symmetric, elastic wave propagation problems. Such solutions are very convenient starting points for approximate studies, in that the modes of harmonic wave propagation do not have to be known exactly. The particular approximation to be pursued here, which is accurate in the far-field, is the head of the pulse approximation.

\section{FAR-FIELD APPROXIMATION}

The dominant, early arriving, far-field disturbance is described, for symmetric waves in homogeneous, infinite rods and plates, by the low-frequency, large wave length portion 
of the lowest mode. The same is taken to be true for the case at hand. To obtain the required mode approximation, the following wave number expansions are employed:

$$
\begin{aligned}
& p_{1}^{2}=P_{0}+(i k)^{2} P_{1}+(i k)^{4} P_{2}+\ldots \\
& { }_{j} u_{x}^{1}={ }_{j} u_{x 0}+(i k)^{2}{ }_{j} u_{x 1}+(i k)^{4}{ }_{j} u_{x 2}+\ldots, \quad j=1,2 \text {, } \\
& { }_{j} u_{z}^{1}=(i k){ }_{j} u_{z 1}+(i k)^{3}{ }_{j} u_{z 2}+(i k)^{5}{ }_{j} u_{z 3}+\ldots, \quad j=1,2,
\end{aligned}
$$

where $P_{0}, P_{1}, P_{2}$, etc., are constants and ${ }_{j} u_{x 0^{-}} u_{z 3}$ are at most functions of $z$.

Substituting (32)-(34) into equations such as (10)-(13) and (17), and the continuity conditions at $z=h_{1}$, sets of relations, which are power series in $k$, are obtained. On selectively terminating these series, groups of relations for the determination of the unknowns in (32)-(34) are found. To order $k^{0}$, one gets

$$
\begin{array}{rlrl}
\frac{\mathrm{d}_{j} \sigma_{x z 1}}{\mathrm{~d} z} & =\rho_{j} P_{0 j} u_{x 0}, & & j=1,2, \\
{ }_{j} \sigma_{x z 1} & =\mu_{j} \frac{\mathrm{d}_{j} u_{x 0}}{\mathrm{~d} z}, & & j=1,2, \\
{ }_{2} \sigma_{x z 1} & =0, & & z=h_{2} \\
{ }_{1} \sigma_{x z 1} & ={ }_{2} \sigma_{x z 1}, & z & =h_{1} \\
{ }_{1} u_{x 0} & ={ }_{2} u_{x 0}, & & z=h_{1} \\
{ }_{1} \sigma_{x z 1} & =0, & & z=0 \\
\rho_{1} \int_{0}^{h_{1}}{ }_{1} u_{x 0}^{2} \mathrm{~d} z+\rho_{2} \int_{h_{1}}^{h_{2}}{ }_{2} u_{x 0}^{2} \mathrm{~d} z=1 / 2 .
\end{array}
$$

These equations are satisfied by

$$
\begin{gathered}
P_{0}=0 \\
{ }_{1} u_{x 0}={ }_{2} u_{x 0}=1 / \sqrt{ }\left\{2\left[\rho_{1} h_{1}+\rho_{2}\left(h_{2}-h_{1}\right)\right]\right\} .
\end{gathered}
$$

Proceeding as in [1], it can be shown that the relations to order $k$ and $k^{2}$, which must be considered together to get a determinate system, can be satisfied by

$$
\begin{gathered}
{ }_{1} u_{z 1}=a z, \quad{ }_{2} u_{z 1}=b z+l \\
{ }_{1} u_{x 1}=c z^{2}+m z+d, \quad{ }_{2} u_{x 1}=f z^{2}+q z+g
\end{gathered}
$$

by a suitable choice of the constants $a-g$. The process is somewhat lengthy, and, in the interests of brevity, the values of the constants will not be given here. It can also be shown that the equations to order $k^{3}$ and $k^{4}$ (the terminal stage for the head of the pulse approximation) can be satisfied by $\dagger$

$$
\begin{aligned}
& { }_{1} u_{z 2}=A z^{3}+B z, \quad{ }_{2} u_{z 2}=\Phi z^{3}+Q z^{2}+D z+F \\
& { }_{1} u_{x 2}=G z^{4}+S z^{3}+I z^{2}+T_{1} z+J \\
& { }_{2} u_{x 2}=K z^{4}+V_{1} z^{3}+L z^{2}+W z+M
\end{aligned}
$$

by an appropriate choice of the constants $A-M$.

$\dagger$ This is a departure from the method used in [1], where at this stage the equations of motion were used. The current technique is felt to be more straightforward and more capable of generalization. 
As regards the lowest mode approximation, one finds, after considerable algebra, that

where†

$$
\begin{aligned}
& \rho_{2} P_{1}=\mu_{2} \Gamma \\
& \rho_{2} P_{2}=\mu_{1} h_{1}^{2} \alpha
\end{aligned}
$$

$$
\begin{aligned}
& \Gamma=\frac{2 R_{\rho}}{R_{\rho}\left(1-R_{h}\right)-1}\left[\frac{1-R_{h}}{1-\sigma_{2}}-\frac{1}{R_{\mu}\left(1-\sigma_{1}\right)}\right] \\
& R_{\rho}=\rho_{2} / \rho_{1}, \quad R_{\mu}=\mu_{2} / \mu_{1}, \quad R_{h}=h_{2} / h_{1} \\
& \alpha=\frac{R_{\rho} \Delta_{1}-R_{\mu} \Delta_{2}}{1+R_{\rho}\left(R_{h}-1\right)}-\frac{R_{\mu} \Psi_{2}}{1-2 \sigma_{2}}+\left[\frac{2\left(1-\sigma_{2}\right)}{1-2 \sigma_{2}}-\Gamma\right] R_{\mu} \Psi_{1} \\
& \Delta_{1}=R_{\mu}\left(1-R_{h}^{3}\right)(4 \zeta-\beta)+R_{\mu}\left(1-R_{h}^{2}\right)(3 \xi+\Psi)-R_{\mu}\left(1-R_{h}\right) \Psi_{2}-4 \eta+\gamma_{3}-\gamma_{1} \\
& \Delta_{2}=-\frac{\Psi_{2}}{1-2 \sigma_{2}}+\left[\frac{2\left(1-\sigma_{2}\right)}{1-2 \sigma_{2}}-\Gamma\right] \Psi_{1}-\frac{R_{\rho}}{R_{\mu}\left(1-2 \sigma_{1}\right)} \gamma_{1} \\
& -\left[\frac{2 R_{\rho}\left(1-\sigma_{1}\right)}{R_{\mu}\left(1-2 \sigma_{1}\right)}-\Gamma\right]\left(\Theta-\gamma+\Delta+\Psi_{1}\right) \\
& \Psi_{2}=R_{h}^{2}\left[\frac{\sigma_{2} \Theta}{1-\sigma_{2}}-3 \beta\right]+R_{h}\left[2 \Psi+\frac{\sigma_{2} \Delta}{1-\sigma_{2}}\right]+\frac{\sigma_{2} \Psi_{1}}{1-\sigma_{2}} \\
& \Psi_{1}=\left\{\frac{\sigma_{1}^{2}}{3\left(1-\sigma_{1}\right)^{2}}-\frac{2}{3} R_{\rho} \Theta\left(R_{h}^{3}-1\right)-\frac{2}{3} \gamma-\left[R_{\rho}\left(R_{h}^{2}-1\right)+2\right] \Delta\right. \\
& +\frac{\sigma_{2}^{2} R_{\rho}\left(R_{h}^{3}-1\right)}{3\left(1-\sigma_{2}\right)^{2}}+\frac{R_{\rho}\left(R_{h}-1\right)\left(\sigma_{2}-\sigma_{1}\right)^{2}}{\left(1-\sigma_{2}\right)^{2}\left(1-\sigma_{1}\right)^{2}} \\
& \left.-\frac{R_{\rho} \sigma_{2}\left(R_{h}^{2}-1\right)\left(\sigma_{2}-\sigma_{1}\right)}{\left(1-\sigma_{1}\right)\left(1-\sigma_{2}\right)^{2}}-2(\Theta-\gamma)\right\}[2+2 R \rho(R h-1)]^{-1} \\
& \zeta=\left\lfloor\Gamma-\frac{2\left(1-\sigma_{2}\right)}{1-2 \sigma_{2}}\right\rfloor \frac{\Theta}{12}+\frac{\beta}{4\left(1-2 \sigma_{2}\right)} \\
& \beta=\frac{\sigma_{2}\left(1-2 \sigma_{2}\right)}{12\left(1-\sigma_{2}\right)^{2}}(\Gamma-1)+\frac{\Theta}{6\left(1-\sigma_{2}\right)} \\
& \xi=\left\lfloor\Gamma-\frac{2\left(1-\sigma_{2}\right)}{1-2 \sigma_{2}}\right] \frac{\Delta}{6}-\frac{\Psi}{3\left(1-2 \sigma_{2}\right)} \\
& \Psi=\frac{\left(1-2 \sigma_{2}\right)\left(\sigma_{2}-\sigma_{1}\right)(\Gamma-1)}{4\left(1-\sigma_{1}\right)\left(1-\sigma_{2}\right)^{2}}-\frac{\Delta}{4\left(1-\sigma_{2}\right)} \\
& \eta=\left[\frac{R_{\mu} \Gamma}{R_{\rho}}-\frac{2\left(1-\sigma_{1}\right)}{1-2 \sigma_{1}}\right] \frac{\gamma}{12}+\frac{\gamma_{3}}{4\left(1-2 \sigma_{1}\right)} \\
& \gamma_{3}=\frac{\sigma_{1}\left(1-2 \sigma_{1}\right)}{12\left(1-\sigma_{1}\right)^{2}}\left(\frac{R_{\mu} \Gamma}{R_{\rho}}-1\right)+\frac{\gamma}{6\left(1-\sigma_{1}\right)}
\end{aligned}
$$

† Though the ensuing expressions are quite complicated, no effort has been made to simplify them further, since very little would be gained by this from the view point of digital computation. 


$$
\begin{aligned}
\gamma_{1}= & 3 \gamma_{3}+\left[\frac{R_{\mu} \sigma_{2}\left(1-2 \sigma_{1}\right)}{\left(1-\sigma_{1}\right)\left(1-2 \sigma_{2}\right)}-\frac{\sigma_{1}}{1-\sigma_{1}}\right]\left(\Theta+\Delta+\Psi_{1}\right) \\
& +\frac{R_{\mu}\left(1-\sigma_{2}\right)\left(1-2 \sigma_{1}\right)}{\left(1-\sigma_{1}\right)\left(1-2 \sigma_{2}\right)}\left(2 \Psi-3 \beta-\Psi_{2}\right) \\
2 \Theta= & \frac{1}{R_{\rho}\left(1-R_{h}\right)-1}\left[\frac{2-\sigma_{2}+\sigma_{2} R_{\rho}\left(1-R_{h}\right)}{1-\sigma_{2}}-\frac{2 R_{\rho}}{R_{\mu}\left(1-\sigma_{1}\right)}\right] \\
2 \gamma= & \frac{1}{R_{\rho}\left(1-R_{k}\right)-1}\left[\frac{2 R_{\mu}\left(1-R_{h}\right)}{1-\sigma_{2}}+\frac{R_{\rho}\left(1-R_{h}\right)\left(\sigma_{1}-2\right)-\sigma_{1}}{1-\sigma_{1}}\right] \\
\Delta= & \frac{2 R_{h}}{R_{\rho}\left(1-R_{h}\right)-1}\left[\frac{R_{p}}{R_{\mu}\left(1-\sigma_{1}\right)}-\frac{1}{1-\sigma_{2}}\right]-\frac{\sigma_{2}-\sigma_{1}}{\left(1-\sigma_{1}\right)\left(1-\sigma_{2}\right)}
\end{aligned}
$$

where $\sigma_{1}$ and $\sigma_{2}$ denote Poisson's ratio for materials one and two, respectively.

Equations (35) $(40)$ are sufficient to implement the head of the pulse approximation, which is now applied to the following three basic problems: (i) Longitudinal impact problem. The edge boundary conditions are

$$
\begin{array}{ll}
{ }_{1} u_{x}={ }_{2} u_{x}=u_{0} S(t), & x=0 \\
{ }_{1} \sigma_{x z}={ }_{2} \sigma_{x z}=0, & x=0
\end{array}
$$

where $u_{0}$ is a constant and $S(t)$ denotes the Heaviside unit step function. Also, the plate is taken to be stress free at $z=h_{2}$. (ii) Mixed pressure shock problem. The edge boundary conditions are

$$
\begin{aligned}
{ }_{1} u_{z}={ }_{2} u_{z}=0, & x=0 \\
{ }_{1} \sigma_{x x}={ }_{2} \sigma_{x x}=-\sigma_{0} S(t), & x=0
\end{aligned}
$$

where $\sigma_{0}$ is a constant. Again, the plate is taken to be stress free at $z=h_{2}$. (iii) Line load problem. The boundary conditions at $z=h_{2}$ are taken as

$$
\begin{array}{ll}
{ }_{2} \sigma_{z z}=-L_{0} S(t) \delta(x), & z=h_{2} \\
{ }_{2} \sigma_{x z}=0, & z=h_{2}
\end{array}
$$

where $L_{0}$ is a constant and $\delta$ denotes the delta function

Inserting (41) $-(46)$ into (29), (30) and (31), the boundary terms can be evaluated. Using (19) and retaining only the odd function of $k$ in these terms gives the transformed solution (Laplace and Fourier sine or cosine) to the longitudinal impact problem, whereas the transformed solutions (Laplace and Fourier sine or cosine) to the pressure shock problem stem from the even functions of $k$. The full expressions are retained for the line load problem, but $B_{O N}$ is set equal to zero. In all cases the Laplace transforms can be inverted by residue theory and the Fourier transforms can be inverted by means of the appropriate inversion theorems. Then, applying the head of the pulse approximation (see [20] for details), some 
typical results for the three problems are:

$$
\begin{gathered}
{ }_{1} u_{x}={ }_{2} u_{x}=u_{0}\left(\frac{1}{3}+\int_{0}^{\psi^{\prime}} A i(-\eta) \mathrm{d} \eta\right) \\
\frac{\mu_{11} \varepsilon_{z z}}{\sigma_{0}}=\frac{\sigma_{1} R_{h}}{2\left[1-R_{\mu}\left(1-R_{h}\right)\left(\frac{1-\sigma_{1}}{1-\sigma_{2}}\right)\right]\left[\frac{1}{3}+\int_{0}^{\psi^{\prime}} A i(-\eta) \mathrm{d} \eta\right]} \\
\frac{\mu_{12} \varepsilon_{z z}}{\sigma_{0}}=\frac{\sigma_{2}\left(1-\sigma_{1}\right) R_{h}}{2\left(1-\sigma_{2}\right)\left[1-R_{\mu}\left(1-R_{h}\right)\left(\frac{1-\sigma_{1}}{1-\sigma_{2}}\right)\right]}\left[\frac{1}{3}+\int_{0}^{\psi^{\prime}} A i(-\eta) \mathrm{d} \eta\right] \\
\frac{\mu_{11} \varepsilon_{x x}}{\sigma_{0}}=\frac{\mu_{12} \varepsilon_{x x}}{\sigma_{0}}=-\frac{\left(1-\sigma_{1}\right) R_{h}}{2\left[1-R_{\mu}\left(1-R_{h}\right)\left(\frac{1-\sigma_{1}}{1-\sigma_{2}}\right)\right]}\left[\frac{1}{3}+\int_{0}^{\psi^{\prime}} A i(-\eta) \mathrm{d} \eta\right] \\
\frac{\mu_{11} u_{x}}{L_{0}}=\frac{\mu_{12} u_{x}}{L_{0}}=\frac{\left(1-\sigma_{1}\right)\left[\frac{\sigma_{2}\left(1-R_{h}\right)}{1-\sigma_{2}}-\frac{\sigma_{1}}{1-\sigma_{1}}\right]}{4\left[1-R_{\mu}\left(1-R_{h}\right)\left(\frac{1-\sigma_{1}}{1-\sigma_{2}}\right)\right]}\left[\frac{1}{3}+\int_{0}^{\psi^{\prime}} A i(-\eta) \mathrm{d} \eta\right]
\end{gathered}
$$

where $A i$ denotes the Airy function, $\varepsilon$ strain and

$$
\begin{aligned}
\psi^{\prime} & =\frac{1}{\tau^{4}}\left(\frac{2 \sqrt{ }\left(R_{\rho} R_{\mu} \Gamma\right)}{3 \alpha}\right)^{\frac{1}{3}}\left(-\zeta_{1}+\tau / \frac{R_{\mu} \Gamma}{R_{p}}\right) \\
\tau & =\frac{t}{h_{1}} \sqrt{\left(\frac{\mu_{1}}{\rho_{1}}\right), \quad \zeta_{1}=x / h_{1} .}
\end{aligned}
$$

\section{NUMERICAL RESULTS}

Some general observations should first be made, namely: (a) The horizontal displacements in materials one and two are identical in the longitudinal impact problem. Moreover, the amplitude of the pulse is independent of material properties and the thickness of the layers. However, the periods that arise do depend on these items. (b) The horizontal strains in materials one and two in the pressure shock problem are identical. (c) The horizontal displacements in materials one and two in the line load problem are identical.

Shown in Figs. 2-7 are the results of some sample computations of (47)-(51). Figures 2-6 exhibit the effects of the thickness ratio $R_{h}$ on the various responses at the station $\zeta_{1}=80$. As regards the other parameters, two cases are treated, namely, $\sigma_{1}=\sigma_{2}=0.25$, $R_{\mu}=13.792275, R_{\rho}=1.39$ (case I), which is an example of seismological interest (see Grant and West [21, p. 84]) and $\sigma_{1}=0.20, \sigma_{2}=0.41, R_{\mu}=0.00834711, R_{\rho}=0.451711$ (case II), which is an example treated by Peck and Gurtman [9] and corresponds to layer one being boron and layer two being epoxy. Figure 2 gives the horizontal displacements in case I for the longitudinal impact problem. It is seen that the greater $R_{h}$ the earlier the arrival of the main disturbance. However, $R_{h}$ appears to have very little effect on the periods. Figures 3 and $\mathbf{4}$ show the horizontal strains in the pressure shock problem for cases I and II, respectively. It is seen that the amplitudes and periods decrease with $R_{h}$. For case II, the 


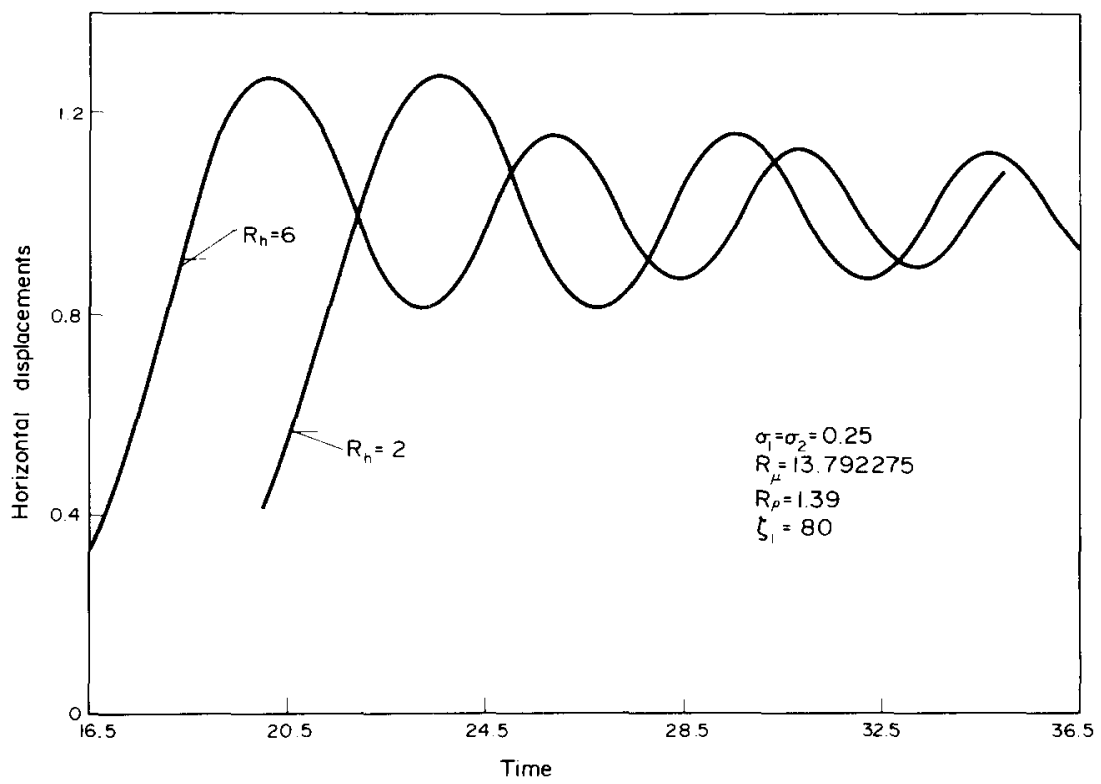

FIG. 2. Horizontal displacements $u_{x} / u_{0}$ vs. time $\left.\tau=t / h_{1} / \sqrt{(} \mu_{1} / \rho_{1}\right)$ in the longitudinal impact problem. Case I.

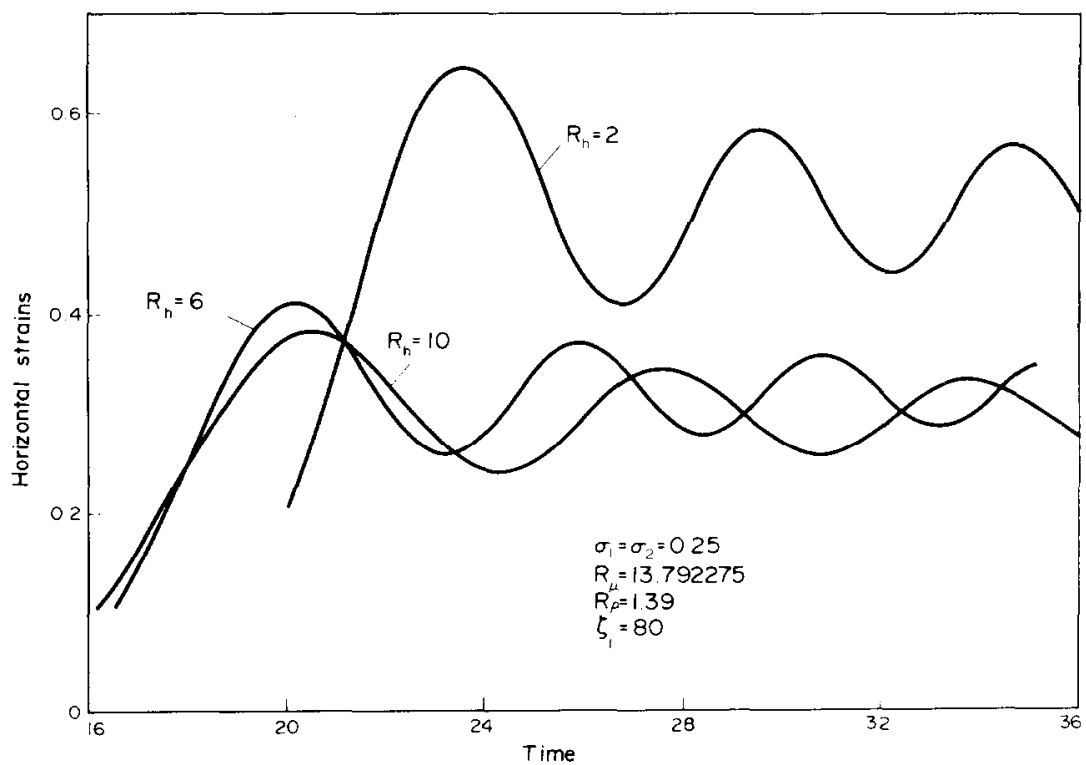

FIG. 3. Horizontal strains $-\mu_{1} \varepsilon_{x x} / \sigma_{0}$ vs. time $\tau=t / h_{1} \sqrt{ }\left(\mu_{1} / \rho_{1}\right)$ in the pressure shock problem. Case I. 


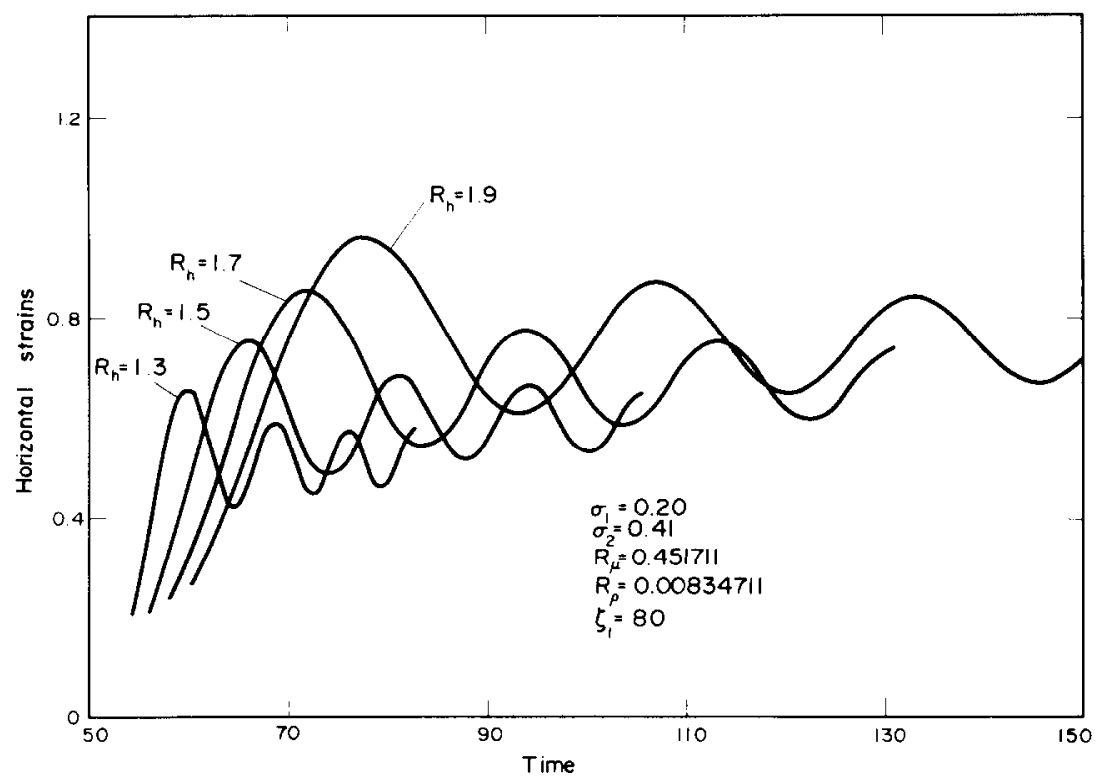

FIG. 4. Horizontal strains $-\mu_{1} \varepsilon_{x x} / \sigma_{0}$ in the pressure shock problem versus time $\tau=t / h_{1} \sqrt{ }\left(\mu_{1} / \rho_{1}\right)$. Case II.

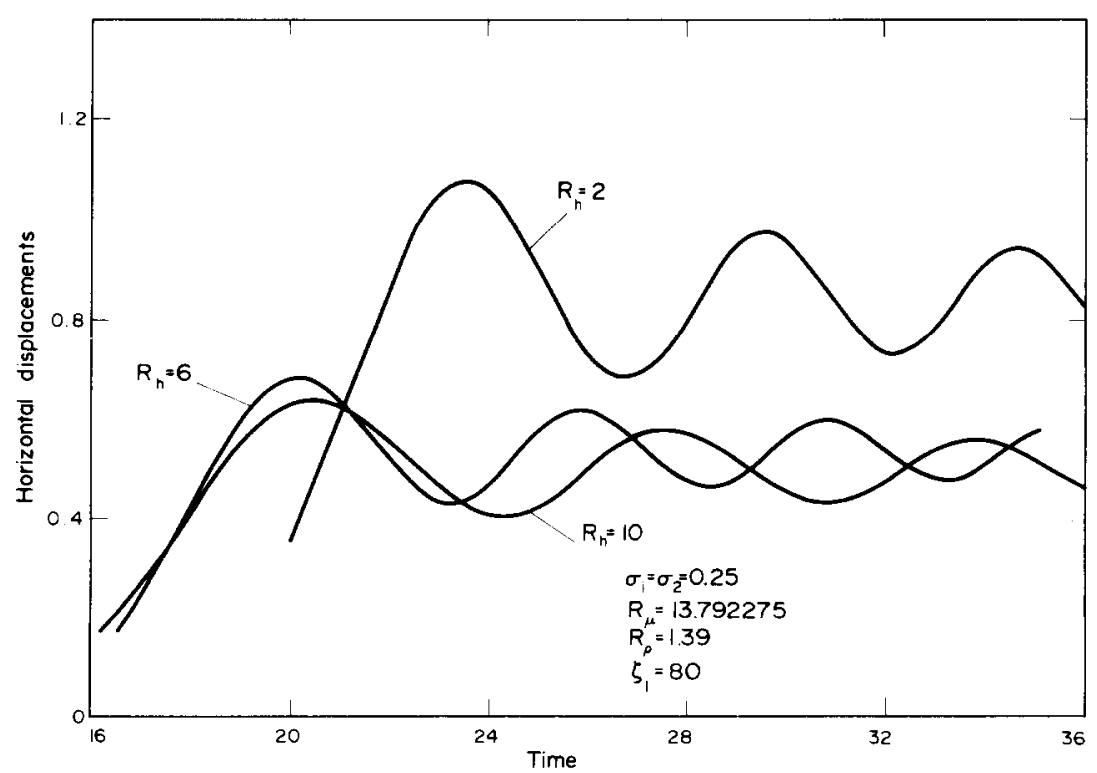

FIG. 5. Horizontal displacements $-\mu_{1} \mu_{x} / L_{0}$ vs. time $\tau=t / h_{1} \sqrt{ }\left(\mu_{1} / \rho_{1}\right)$ in the line load problem. Case I. 


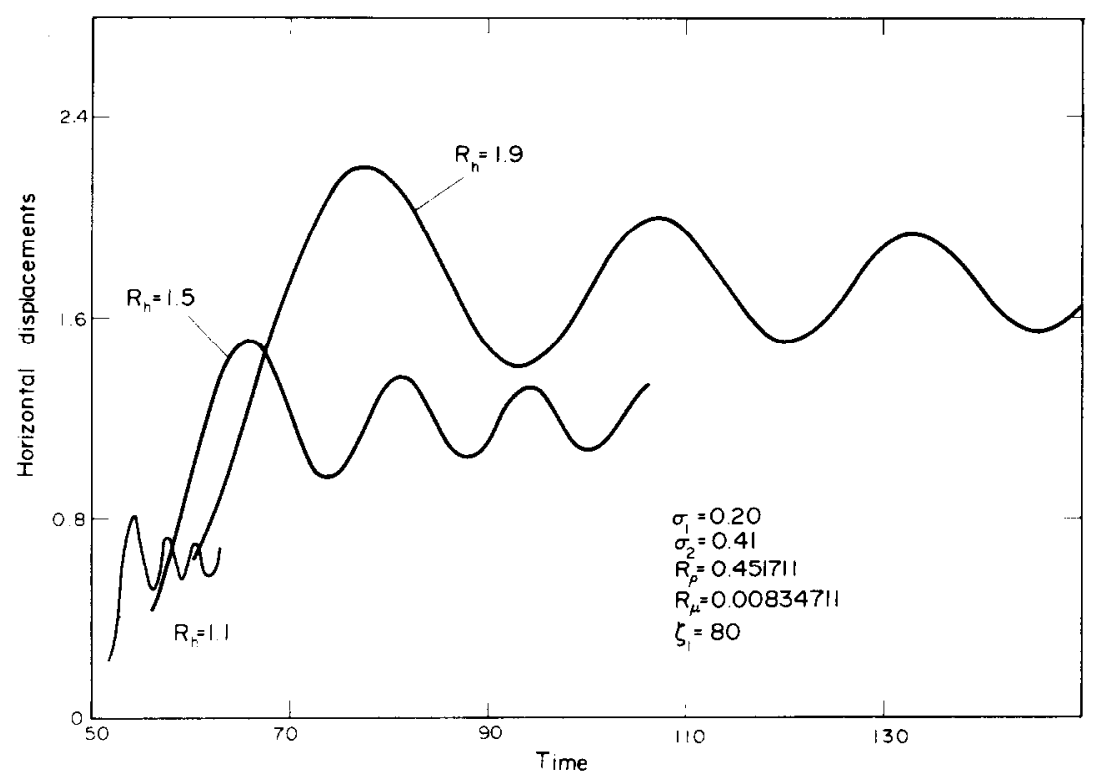

Fig. 6. Horizontal displacements $-\mu_{1} u_{x} / u_{0}$ vs. time $\tau=t_{1} / h_{1} \sqrt{ }\left(\mu_{1} / \rho_{1}\right)$. Case II.

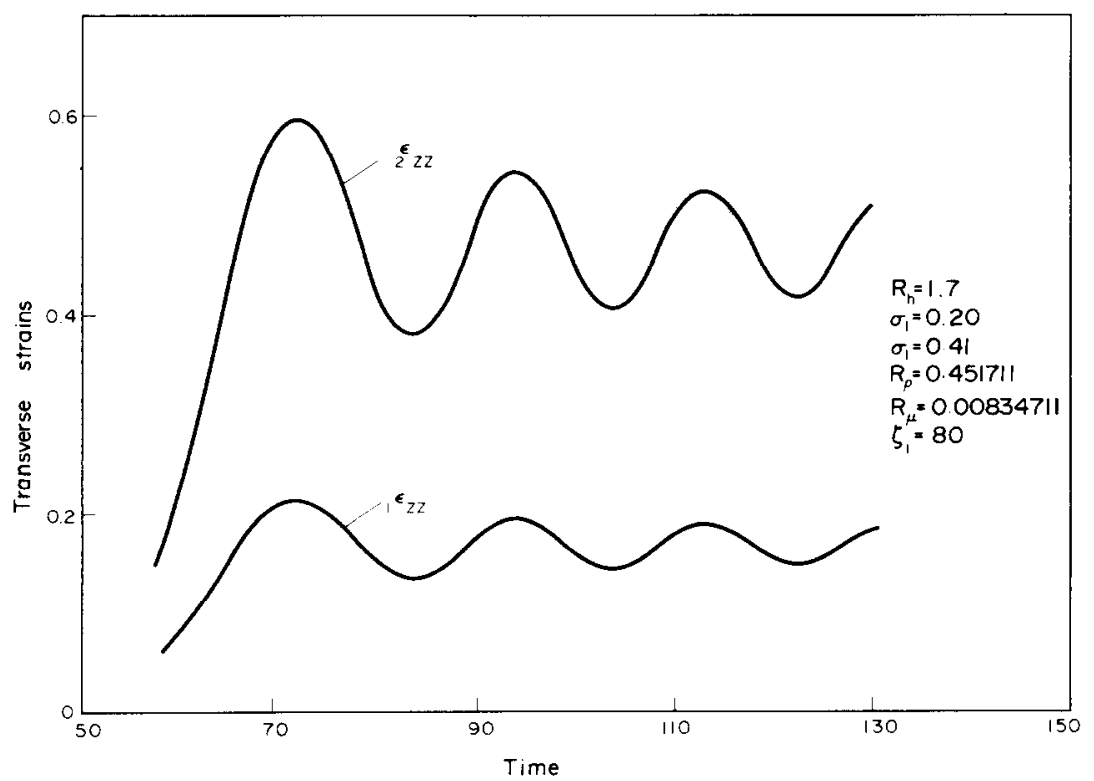

FIG. 7. Transverse strains $\mu_{11} \varepsilon_{z z} / \sigma_{0}$ and $\mu_{12} \varepsilon_{z z} / \sigma_{0}$ vs. time $\tau=t / h_{1} \sqrt{ }\left(\mu_{1} / \rho_{1}\right)$ in the pressure shock problem. Case II. 
situation is reversed. The larger $R_{h}$, the greater are the amplitudes and periods. Figures 5 and 6 give the horizontal displacements in the line load problem for cases I and II, respectively. The main features that emerge are that in case I the greater $R_{h}$, the smaller the amplitudes but the larger the periods, whereas for case II, both amplitudes and periods increase with $R_{h}$.

Shown in Fig. 7 are the transverse strains for case II in the pressure shock problem at the station $\zeta_{1}=80$. It is seen that the strains in layer two are considerably larger than those in layer one.

\title{
REFERENCES
}

[1] R. A. Scott, Transient elastic waves in an inhomogeneous layer. Bull. Seism. Soc. Am. 60, 383 (1970).

[2] M. Newlands, The disturbance due to a line source in a semi-infinite elastic medium with a single surface layer. Phil. Trans. R. Soc., Lond. A245, 213 (1952).

[3] C. L. Pekeris, Z. Alterman and E. Abramovici, Propagation of an $S H$-torque pulse in a layered solid. Bull. Seism. Soc. Am. 53, 39 (1963).

[4] C. L. Pekeris, Z. Alterman and F. Abramovici, Propagation of a compressional pulse in a layered solid. Rev. Geophys. 3, 25 (1965).

[5] M. Mitra, An $S H$-point source in a half-space with a layer. Bull. Seism. Soc. Am. 53, 1031 (1963).

[6] S. J. LASter, J. G. Foreman and A. F. Linville, Theoretical investigation of modal seismograms for a layer over a half-space. Geophysics 30, 571 (1965).

[7] M. Mitra, On $S H$-point source in a two-layered half space. Gerl. Beit. Z. Geophys. 74, 41 (1965).

[8] L. E. Voflker and J. D. Achenbach, Stress waves generated by transverse forces. J. acoust. Soc. Am. 46, 1213 (1969).

[9] J. C. Peck and G. A. Gurtman, Dispersive pulse propagation parallel to the interfaces of a laminated composite. J. appl. Mech. 36, 479 (1969).

[10] C. Sve, Propagation of a shear pulse parallel to the interface of a periodically laminated medium. J.appl. Mech. 37, 203 (1970).

[11] J. D. Achenbach, C. T. Sun and G. Herrmann, On the vibrations of a laminated body. J. appl. Mech. 35, 689 (1968).

[12] R. A. Scotr, Transient Anisotropic Waves in Bounded Elastic Media, Wave Propagation in Solids, ASME Monograph, p. 71 (1969).

[13] R. L. Rosenfeld and J. Miklowitz, Elastic wave propagation in rods of arbitrary cross-section. J. appl. Mech. 32, 290 (1965).

[14] J. D. AChenbaCh, An asymptotic method to analyze the vibration of an elastic layer. J. appl. Mech. 36, 65 (1969).

[15] J. D. ACHENBaCH and S. J. FANG, Asymptotic analysis of the modes of wave propagation in a solid cylinder. J. acoust. Soc. Am. 47, 1282 (1970).

[16] H. Reismann, On the forced motion of elastic solids. Appl. Sci. Res. 18, 156 (1967).

[17] Y. C. LeE and H. ReISmann, Dynamics of rectangular plates. Int. J. Engng Sci. 7, 93 (1969).

[18] C.-H. Wu and R. Plunkett, On the solutions of plates, strips, rods and cylinders subjected to arbitrary dynamic edge load. Siam J. appl. Math. 15, 107 (1967).

[19] M. H. Conre, Dynamic vibrations and stresses in composite elastic plates. J. acoust. Soc. Am. 46,1175 (1969).

[20] R. A. ScotT and J. MikLowitz, Transient elastic waves in anisotropic plates. J. appl. Mech. 34, 104 (1967).

[21] F. S. GRANT and G. F. WEST, Interpretation Theory in Applied Geophysics. McGraw-Hill (1965).

(Received 18 June 1971 ; revised 6 October 1971)

\begin{abstract}
Абстракт-Представляются формальные решения, выраженные зависимостями распространения гармонической волны, для широкого класса нестационарных, симметрических, упругих волн в трехслойных пластинках. Дается приближение самого малого вида колебаний. Используя этот факт и формальные решения, даются выражения для удаленных полей трех спеуифических задач. Обсуждаются численно этие приближенные выражения для двух специфических случаев.
\end{abstract}

